\title{
F. W. Gehring: Preface
}

\section{Olli Martio}

Published online: 27 August 2014

(C) Springer-Verlag Berlin Heidelberg 2014

This volume of Computational Methods and Function Theory is dedicated to the memory of Frederick W. Gehring.

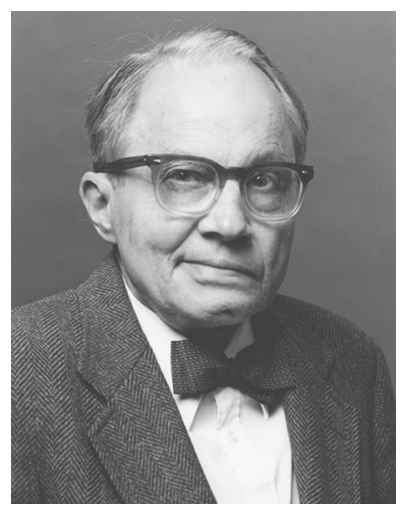

The articles by P. Duren and G. Martin portray his life and achievements. A conference to celebrate his memory was organized at the University of Helsinki, August 11-12, 2013, and the conference title "A quasiconformal life: Celebration of the legacy and work of F. Gehring" summarizes the life-long interest of F. Gehring. Many contributors to this volume, as well as his students and co-authors, participated at the conference.

F. W. Gehring had a special connection with Finland. As a Fulbright grantee he arrived in Finland interested in the normal families of meromorphic functions but the Finnish function theoretic school, led by O. Lehto and K. Virtanen in particular,

O. Martio $(\square)$

Finnish Academy of Science and Letters, Mariankatu 5, 00170 Helsinki, Finland e-mail: olli.martio@acadsci.fi 
had switched their attention to quasiconformal maps. In the 1958-1959 seminar F. W. Gehring learned both the current theory of quasiconformal maps and the Finnish language. Although the origin of quasiconformal maps stems from complex analysis, the methods of real analysis were to play the major role in the theory of quasiconformal maps in space. F. W. Gehring was the right person to lead this development. His thesis and early studies were in real analysis.

Later he and many of his students spent several semesters and participated in many conferences in Finland. (I remember very well two meetings, the Nordic Summer School in Helsinki 1969, where the main speakers were L. Ahlfors, F. W. Gehring and A. Selberg, and the workshop "Domains and Mappings" at the University of Jyväskylä in 1989. The Nordic Summer School was the first conference I had ever organized. The second conference was organized when the studies of uniform domains and their connections to quasidisks were about to reach their culmination). From these stays a life-long co-operation and friendship with Finnish mathematicians emerged. K. Astala, J. Heinonen, A. Hinkkanen, M. Vuorinen, J. Väisälä and I were among those Finnish mathematicians who had the privilege to work with F. W. Gehring and enjoy his hospitality in Ann Arbor. In the article [1] O. Lehto gives a more detailed account of F. W. Gehring's contacts with Finland.

F. W. Gehring had also close connections to other Nordic countries. He visited the Mittag-Leffler Institute in Stockholm several times. He and L. Carleson addressed the higher integrability problem of the derivatives of space quasiconformal mappings and the extension problem of a quasiconformal mapping from the three-dimensional Euclidean space to four dimensions during the special semester "Quasi-conformal Mappings" at the Institute in 1972. Both attempts were successful. Gehring's solution, based on the reverse Hölder inequality, started a new era in the regularity theory for solutions of linear and non-linear partial differential equations. He was also the Onsager Professor at the Norwegian Institute of Technology (NTH) in 1995 where he worked with K. Hag to finish their book on his favorite subject, quasidisks.

Many articles in this volume originate from the papers of F. W. Gehring, and I hope that the contributors as well as his students continue the legacy of F. W. Gehring who was always looking for new connections and applications of quasiconformal maps.

The editors thank the CMFT journal for publishing this memorial volume and the journal office for co-operation. We also thank Eero Astala for letting us to use his artwork for the cover page and the referees for their work.

\section{Reference}

1. Lehto, O.: Fred Gehring and Finnish Mathematics, Quasiconformal mappings and analysis (Ann Arbor, MI, 1995), pp. 19-32. Springer, Heidelberg (1998) 